\title{
Suppression of Gingival Inflammation Induced by Porphyromonas gingivalis in Rats by Leupeptin
}

\author{
Sizuo Kitano ${ }^{1, *}$, Kenji Irimura ${ }^{2}$, Toru Sasaki ${ }^{1}$, Naoko $\mathrm{Abe}^{3}$, Atsuyo $\mathrm{Baba}^{3}$, Yoichiro Miyake ${ }^{4}$, \\ Nobuhiko Katunuma ${ }^{5}$ and Kenji Yamamoto ${ }^{3}$ \\ ${ }^{1}$ Pharmacology Research Laboratory, ${ }^{2}$ Drug Safety Research Laboratory, Tokushima Research Center, Taiho Pharmaceutical Co., Ltd., \\ 224-2, Ebisuo, Hiraishi, Kawauchi-cho, Tokushima 771-0194, Japan \\ ${ }^{3}$ Department of Pharmacology, Faculty of Dentistry, Kyushu University, Higashi-ku, Fukuoka 812-8582, Japan \\ ${ }^{4}$ Department of Microbiology, Tokushima University School of Dentistry, 3-18-15, Kuramoto-cho, Tokushima 770-8504, Japan \\ ${ }_{5}^{5}$ Institute for Health Sciences, Tokushima Bunri University, 180, Bouji, Nisihama, Yamashiro-cho, Tokushima 770-8514, Japan
}

Received September 4, 2000 Accepted October 26, 2000

\begin{abstract}
In this study, we developed a procedure to produce gingivitis in rats by inoculation of Porphyromonas gingivalis and studied the contribution of the bacterial cysteine proteinases, Arg-gingipain (Rgp) and Lys-gingipain (Kgp), to the pathology in the gingiva. To adhere the bacterium to periodontal tissues, a cotton thread was inserted between the first and second molar of right maxillary sites of rats. Rats in group A were administered with vehicle alone after bacterial (strain W83) inoculation. In group B, the bacteria were inoculated in combination with leupeptin, a potent inhibitor of Rgp and Kgp, and then leupeptin alone was administered the week after. Rats in group C were administered leupeptin for 6 weeks after bacteria inoculation. All left maxillary gingiva in three groups showed no inflammatory changes. Right maxillary gingiva of group A showed most of the clinical landmarks of gingivitis. Leupeptin exhibited only a little inhibitory effect on this gingivitis in group B, whereas it had a strong inhibitory effect on the inflammation in group C. These results suggest that $P$. gingivalis-induced gingivitis is attributable to Rgp and Kgp and that leupeptin is more effective in the late phase than the early stage of gingivitis.
\end{abstract}

Keywords: Arg-gingipain, Lys-gingipain, Gingivitis, Leupeptin, Porphyromonas gingivalis

Porphyromonas gingivalis, a black-pigmented, Gramnegative anaerobe, is widely implicated as an important etiologic agent in certain forms of periodontitis, particularly adult periodontitis in humans (for reviews, see refs. $1-5$ ). The virulence factors of $P$. gingivalis have been studied in some detail (for reviews, see refs. 2-5). These include fimbriae and lectin-type adhesions, a polysaccharide capsule and lipopolysaccharide, hemagglutinins and various hydrolytic enzymes. Among these, proteolytic enzymes may play a major role in $P$. gingivalis virulence, since they have the ability to degrade host tissues and immune response mediators, toxic metabolites and cellular constituents, and adherence factors that promote colonization. In addition, these enzymes may be crucial for providing heme and amino acids that are essential for the growth and proliferation of this asaccharolytic organism (6). Most of the proteinases that have been referred to as trypsin-like enzymes are found to be due to two individual cysteine proteinases,

*Corresponding author. FAX: +81-88-665-6554

E-mail: shi-kitano@taiho.co.jp
Arg-gingipain (Rgp) and Lys-gingipain (Kgp) (7). These two enzymes represent a new class of the cysteine proteinase family, since they have novel structural features distinct from other known cysteine proteinases $(2-5)$. Recent studies suggest that gingipains are directly responsible for neutrophil accumulation (8), disruption of neutrophil function $(9-11)$, bleeding tendency $(12-14)$ and gingival fluid production (15). The periodontopathogenic importance of gingipains is further justified by that fact that $P$. gingivalis mutants devoid of the activities of Rgp and/or Kgp exhibit the marked reduction of the above virulence and adhesion /hemagglutinin activity $(6,10,14,16,17)$. In addition, the mutant defective in both Rgp and/or Kgp activities shows no ability to acquire peptides/amino acids and heme essential for bacterial growth (6) and less virulence in a mouse model system (16). Therefore, it is tempting to speculate that $P$. gingivalis virulence could be attenuated by inhibition of gingipains with proteinase inhibitors.

The use of animal models in the study of periodontal inflammation (gingivitis and periodontitis) is critical for elucidating the direct association of gingipains with 
$P$. gingivalis-induced pathologic processes. In mouse models, oral infection with $P$. gingivalis can induce alveolar bone loss (18). To date, however, there have been no reports of association of gingipains with $P$. gingivalis-induced pathology in an in vivo model. The purpose of the present study was to clarify whether gingipains are responsible for gingival inflammation induced by oral infection of rats with $P$. gingivalis and to determine the potential for leupeptin, a potent inhibitor of both Rgp and Kgp, to inhibit the onset and progression of $P$. gingivalis-mediated gingival inflammation.

\section{MATERIALS AND METHODS}

\section{Bacteria}

Four strains of $P$. gingivalis, ATCC33277, W50, 1121 and W83, were used in this study. W83 was cultured in liquid brain-heart-infusion broth (Difco Lab., Detroit, MI, USA) supplemented with $5 \mu \mathrm{g} / \mathrm{ml}$ of hemin and $0.5 \mu \mathrm{g} / \mathrm{ml}$ of menadione (BHK-HK medium). One $\mathrm{ml}$ of a stock culture of $P$. gingivalis $\mathrm{W} 83$ stored at $-80^{\circ} \mathrm{C}$ was inoculated to $50 \mathrm{ml}$ of BHK-HK medium and cultured in an anaerobic condition (Anaerobic System Model 1025; Forma Scientific Inc., Marietta, OK, USA) at $37^{\circ} \mathrm{C}$ for 2 days. Then, $51 \mathrm{ml}$ of the culture was inoculated to $1000 \mathrm{ml}$ of new BHK-HK medium and then cultured for an additional 2 days to obtain $P$. gingivalis W83 in logarithmic phase. Proteolytic activities of Rgp and Kgp in the culture supernatants were determined by the use of synthetic substrates, carbobenzoxyPhe-Arg-MCA (Z-Phe-Arg-MCA; Peptide Institute, Inc., Minoh) (9) and Z-His-Glu-Lys-MCA (19). Briefly, $1 \mathrm{ml}$ of the reaction mixture contained various amounts of culture supernatant, $10 \mathrm{mM}$ substrate and $5 \mathrm{mM}$ cysteine in $20 \mathrm{mM}$ sodium phosphate buffer ( $\mathrm{pH}$ 7.5). After incubation at $40^{\circ} \mathrm{C}$ for $10 \mathrm{~min}$, the reaction was terminated by addition $1 \mathrm{ml}$ of $10 \mathrm{mM}$ isoacetic acid (pH 5.0), and the released 7-amino-4-metylcoumarin was measured at $460 \mathrm{~nm}$ (excitation at $380 \mathrm{~nm}$ ) by a fluorescence spectrophotometer. ATCC33277, W50 and 1121 were cultured and proteolytic activities in their culture supernatants were measured with the same procedure used for W83.

To judge whether the four strains of $P$. gingivalis form black-pigmented colonization or not, the strain was inoculated to Brucella agar (Difco Lab.) plates and cultured in an anaerobic chamber for 3 days at $37^{\circ} \mathrm{C}$.

\section{Antibacterial activity of leupeptin against $P$. gingivalis}

Antibacterial activity of leupeptin against $P$. gingivalis ATCC33277 was measured by a broth microdilution method according to the approved standard of the Japan Society of Chemotherapy (20). A bacterial suspension $\left(1.89 \times 10^{6} \mathrm{CFU} / \mathrm{ml}\right)$ of ATCC33277 was inoculated into the wells of the microdilution plates containing $0.1 \mathrm{ml}$ of
BHI-HK medium, ABCM medium (anaerobic bacterial culture medium; Eiken Chemical Co., Ltd., Tokyo), or modified GAM medium (Nissui Seiyaku Co., Ltd., Tokyo). These cultures were incubated in the anaerobic chamber for 24 or $48 \mathrm{~h}$ at $37^{\circ} \mathrm{C}$, and minimum inhibitory concentration (MIC) was determined. Minomycin hydrochloride, erythromycin, ampicillin, cefaclor, ofloxacin and bestatin were purchased from Sigma Co., Ltd. (St. Louis, MO, USA). Leupeptin, chymostatin, antipain and E-64 were purchased from Peptide Institute, Inc.

\section{Animals and thread insertion}

Before the thread insertion, 20 specific pathogen-free (SPF) Wistar male rats (5-week-old; Clea Japan Inc., Tokyo) were fed with the ordinary solid diet (CE-2; Oriental Yeast Co., Ltd., Suita). After thread insertion, they were fed a powdered diet (Diet 2050, Oriental Yeast Co.) containing $28 \%$ skim milk, $28 \%$ sucrose, $28 \%$ cornstarch, $6 \%$ wheat powder, $4 \%$ brewer's yeast, $3 \%$ alphameal, $1 \%$ liver powder, $2 \% \mathrm{NaCl}$. Rats were housed in steal cages at each group.

The animals were administered ad lib with $100 \mu \mathrm{g} / \mathrm{ml}$ of kanamycin (Sigma) and $100 \mu \mathrm{g} / \mathrm{ml}$ of ampicillin via the drinking tap water for four days (day 5 to day 2) before the thread insertion. At day 0 (6-week-old), the animals were anesthetized with intraperitoneal pentobarbital sodium $(50 \mathrm{mg} / \mathrm{kg})$. The cotton thread (Katan 50) having knots at the both ends was inserted between the first and second molars of right maxillary sites (Fig. 1). At day 3, the animals were divided into 3 groups (A, B and C).

The cells of $P$. gingivalis W83 in logarithmic phase were collected and adjusted to a cell count of $1 \times 10^{10} \mathrm{CFU} / \mathrm{ml}$ with BHI-HK medium containing $0.3 \mathrm{mg} / \mathrm{ml}$ of thioglycolate sodium. In group A, P. gingivalis W83 $(0.2 \mathrm{ml}$, $10^{10} \mathrm{CFU} / \mathrm{ml}$ ) was inoculated into rat mouths four times at 2-h intervals per day for 5 consecutive days (Fig. 2). Two days after the inoculation, $0.2 \mathrm{ml}$ of deionized water was administered similarly as a vehicle five times at 2-h intervals per day for 5 days. Between the third week and 7 th week, the rats were administered in the same manner in second week. In group B, P. gingivalis W83 was inoculated in combination with leupeptin $(0.2 \mathrm{ml}, 1 \mathrm{mg} / \mathrm{ml})$ four times at 2-h intervals per day for 5 consecutive days. Two days after the inoculation, leupeptin alone was applied to the mouth of each rat five times at 2-h intervals per day for 5 days. Then, deionized water was administered similarly as a vehicle five times at the same intervals per day for 5 consecutive days between the third week and 7 th week. In group C, $P$. gingivalis W83 was inoculated in the same manner as described for group A. Two days after the inoculation, leupeptin was administered into their mouths five times at 2-h intervals per day for 5 days. Between the third week and 7 th week, the rats were administered in the same manner as 


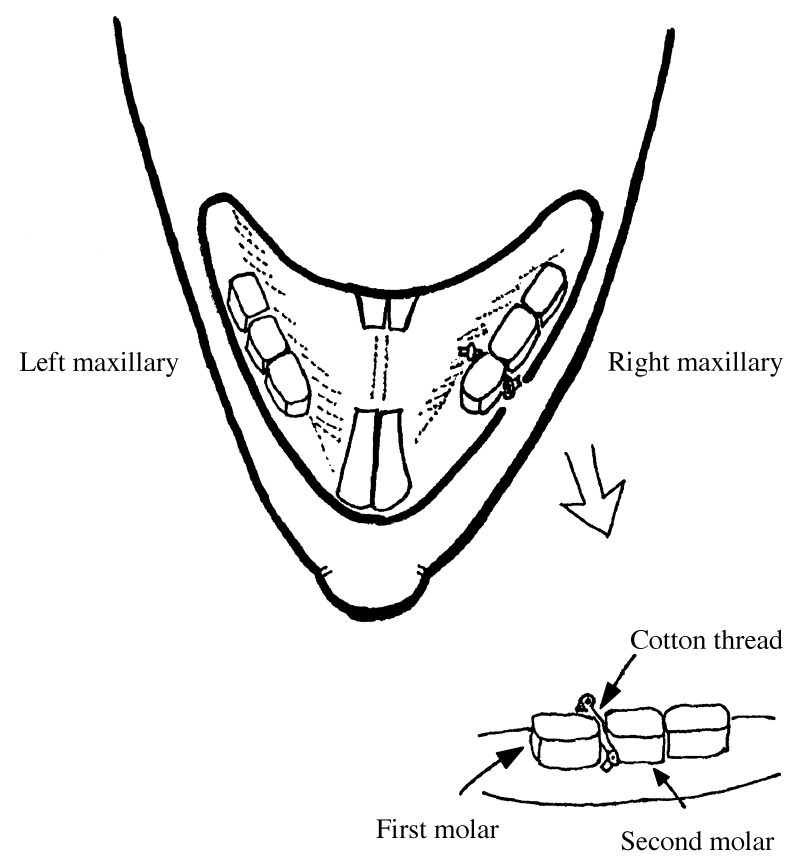

Fig. 1. Cotton thread insertion between the first and second molar of rat right maxillary. in the second week.

\section{Histopathological examination}

Seven weeks after the first bacterial inoculation, animals were sacrificed for histopathological examination. The maxillary bilateral first and second molar samples with periodontal tissues were cut out. The samples were fixed in $20 \%(\mathrm{v} / \mathrm{v})$ buffered formalin for 7 days, defatted with ethanol for 3 days, decalcified with $25 \%$ (v/v) formic acid solution containing $10 \%(\mathrm{w} / \mathrm{v})$ sodium citrate for 4 days and refixed in $10 \%(\mathrm{v} / \mathrm{v})$ buffered formalin. The samples were sectioned to obtain the mesio-distal cross section of interproximal periodontal tissue between the maxillary first and second molars, stained with the hematoxylin-eosin staining solution (Sigma) and examined by a light microscopy.

As the parameters of gingival inflammation, the histopathological changes at the interproximal periodontal tissue were focused on the inflammatory changes (neutrophil infiltration, dilated blood capillaries and focal hemorrhage) and epithelial changes (proliferation, erosion, edema, desquamation and formation of gingival pocket). These changes were evaluated with the following grading codes: - , no changes; +, slight; ++, moderate; +++, severe. The

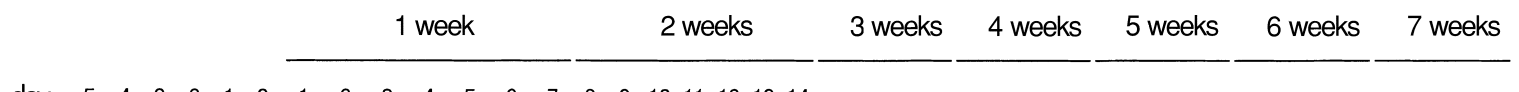

day $\begin{array}{rlllllllllllllllllll}-5 & -4 & -3 & -2 & -1 & 0 & 1 & 2 & 3 & 4 & 5 & 6 & 7 & 8 & 9 & 10 & 11 & 12 & 13 & 14\end{array}$

Vehicle

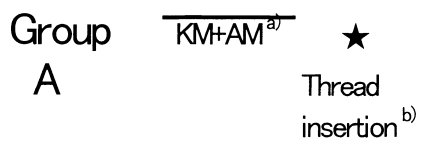

T444

P.G. inoculation ${ }^{\text {) }}$

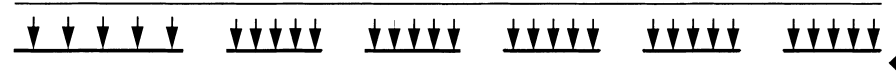

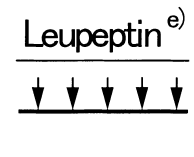

P.G inoculation

Thread insertion

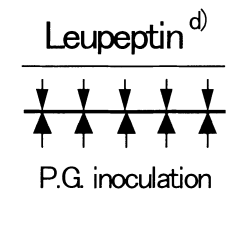

\begin{tabular}{|c|c|c|c|c|}
\hline & & Vehicle & & \\
\hline ivtvi & 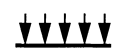 & 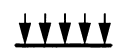 & 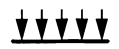 & ivtru \\
\hline
\end{tabular}

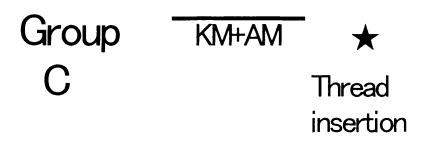

Leupeptin $^{\text {e) }}$

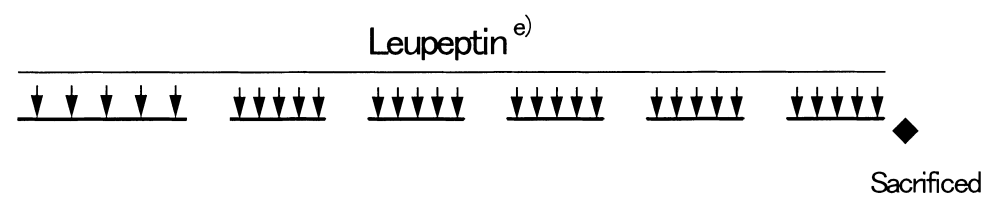

Fig. 2. Experimental design. See Materials and Methods. ${ }^{\mathrm{a}} \mathrm{KM}+\mathrm{AM}$ : the animals were administered ad lib with $100 \mu \mathrm{g} / \mathrm{ml}$ of kanamycin and $100 \mu \mathrm{g} / \mathrm{ml}$ of ampicillin via the drinking tap water for four days (day -5 to day -2 ), ${ }^{\mathrm{b}}$ Thread insertion: the cotton thread (Katan 50) having knots at the both ends was inserted between the first and second molar of the right maxillary site, ${ }^{\mathrm{c}} \mathrm{P}$.G. inoculation: $0.2 \mathrm{ml}$ of cell suspension $\left(10^{10} \mathrm{CFU} / \mathrm{ml}\right)$ of $P$. gingivalis $\mathrm{W} 83$ was inoculated into the rat's mouth four times at 2-h intervals per day (day 3 to day 7), ${ }^{\mathrm{d}}$ Leupeptin: $0.2 \mathrm{ml}$ of leupeptin $(1 \mathrm{mg} / \mathrm{ml})$ was coadministered orally with $P$. gingivalis cell suspension four times at 2-h intervals per day, ${ }^{\text {e) }}$ Leupeptin: $0.2 \mathrm{ml}$ of leupeptin $(1 \mathrm{mg} / \mathrm{ml})$ was administered orally five times at $2-$ $\mathrm{h}$ intervals per day, ${ }^{\mathrm{f}}$ Sacrificed: 7 weeks after the first bacterial inoculation, animals were sacrificed for histopathological examination. 
number of evaluated cases were 6, 7 and 5 in group A, group $\mathrm{B}$ and group $\mathrm{C}$, respectively.

\section{Statistical analyses}

Differences of experimental samples against the sample of right maxillary site of group A (thread-inserted and vehicle-treated molars) as the basal sample were tested by the unpaired Student's $t$-test by use of the soft $\left(\mathrm{SAS}^{\circledR}\right.$ system Version 6.12; SAS Institute Inc., Cary, NC, USA) with $P=0.05$ as the level of significance.

\section{RESULTS}

\section{Profile of $P$. gingivalis strains}

The third day and fourth day culture supernatants from P. gingivalis ATCC33277, W50, 1121 and W83 strains were assayed for proteolytic activities of Rgp and Kgp. The W83 strain and the W50 strain showed more proteolytic activities of both enzymes than the ATCC33277 strain. The 1121 strain showed little proteolytic activity. In addition, the W83 and 1121 strains produced black-pigmented colonies more strongly than the ATCC33277 stain when inoculated on the laked sheep blood agar plates. The W50 strain lacked the ability to produce black-pigmented colony. Taken together, our preliminary experiments indicated the inability of the ATCC33277 to induce experimental gingivitis in a Wistar rat model. Therefore, the strain W83 was selected as the inoculum to build up the experimental gingival inflammation in rats.

Table 1. Antibacterial activities of antibiotics and proteinase inhibitors against $P$. gingivalis ATCC 33277 in some media

\begin{tabular}{lccc}
\hline & \multicolumn{3}{c}{ MIC $(\mu \mathrm{g} / \mathrm{ml})$} \\
\cline { 2 - 4 } Compound & BHI-HK & ABCM & modified GAM \\
\hline Minocycline & 6.25 & 0.39 & 0.006 \\
Erythromycin & 0.39 & 0.39 & 0.2 \\
Ampicillin & 0.39 & 0.78 & 0.1 \\
Cefaclor & 3.13 & 6.25 & 0.78 \\
Ofloxacin & 6.25 & 6.25 & 0.39 \\
Bestatin & 3.13 & 3.13 & 1.56 \\
Leupeptin & $>100$ & $>100$ & $>100$ \\
Chymostatin & $>100$ & $>100$ & $>100$ \\
Antipain & $>100$ & $>100$ & $>100$ \\
E-64 & $>100$ & $>100$ & $>100$ \\
\hline
\end{tabular}

MICs were determined by the broth microdilution method in $0.1 \mathrm{ml}$ of BHI-HK medium, ABCM medium or modified GAM medium according to the approved standard of the Japan Society of Chemotherapy. Microdilution plates received an inoculum of $1.89 \times 10^{6} \mathrm{CFU} /$ well of P. gingivalis ATCC33277 cells. The MIC was defined as the lowest antimicrobial concentration that inhibited visible growth in BHI-HK medium and in ABCM medium $24 \mathrm{~h}$ after and in modified GAM medium $48 \mathrm{~h}$ after the anaerobic condition at $37^{\circ} \mathrm{C}$.
Antibacterial activity of leupeptin against $P$. gingivalis

All antibiotics tested (minocycline, erythromycin, ampicillin, cefaclor and ofloxacin) could exhibit strong antibacterial activities in three different media against $P$. gingivalis ATCC33277 (Table 1). Bestatin, an aminopeptidase inhibitor, exhibited relatively strong antibacterial activity, but leupeptin, chymostatin, antipain and E-64 had little or no antibacterial activity (MIC $>100 \mu \mathrm{g} / \mathrm{ml}$ ). These data indicate that leupeptin is not cytotoxic for $P$. gingivalis.

\section{Histological examination for thread inserted and non- inserted periodontal tissues}

The left maxillary sites of group A (non-inserted sites) showed no or only slight changes in all the sites examined. The same changes as group A were observed in the left maxillary sites of group B and C. On the other hand, the right maxillary sites (thread-inserted site) of group A showed clinical landmarks of gingival inflammation (Table 2 and Fig. 3). In group A, a number of neutrophils were on the surface of the gingival, having presumably migrated through the sulcus. Dilated blood capillaries and swelling of fibroblasts in lamina propria, proliferation, erosion and edema in stratified squamous epithelium were apparent. Desquamation of junctional epithelium was also observed. Formation of gingival pocket was observed in two animals of six. However, there was no evidence of dental colonal caries and alveolar bone loss.

\section{Effect of oral leupeptin on gingival inflammation induced by $P$. gingivalis}

The histological sections form the thread-inserted sites (right) of group B, as compared with those of group A, showed a significantly lower grade for edema in stratified squamous epithelium (Table 2 and Fig. 3). However, there were no significant differences between these two groups with regard to other inflammatory signs such as neutrophil infiltration, dilated blood capillaries in lamina propria, and proliferation, erosion and desquamation of junctional epithelium in stratified squamous epithelium. Dental colonal caries and alveolar bone loss also were not evident in group B. On the other hand, the histological sections from the thread-inserted site of group $\mathrm{C}$ showed a significantly lower grade for erosion in stratified squamous epithelium and the tendency of lower grades for neutrophil infiltration $(P=0.058)$, for dilated blood capillaries $(P=0.104)$, for proliferation and edema of stratified squamous epithelium ( $P=0.058$ and $P=0.059$ respectively) (Table 2 and Fig. 3 ).

These data indicate that leupeptin administered in the early stage (two weeks after $P$. gingivalis inoculation) of $P$. gingivalis-induced gingival inflammation only has a moderate inhibitory effect on the onset and progression of gingival inflammation such as adherence and colonization of $P$. gingivalis to periodontal tissue. In contrast, continu- 
Table 2. Histolgical changes of gingival tissues of rats implanted with oral $P$. orphyromonas gingivalis

\begin{tabular}{|c|c|c|c|c|c|c|c|c|c|c|c|c|c|}
\hline \multirow{3}{*}{ Site/Findings } & \multicolumn{7}{|c|}{ Group A $(n=6)$} & \multirow{2}{*}{\multicolumn{3}{|c|}{$\begin{array}{c}\text { Group B }(\mathrm{n}=7) \\
\text { Right inserted }\end{array}$}} & \multirow{2}{*}{\multicolumn{3}{|c|}{$\begin{array}{c}\text { Group C }(\mathrm{n}=5) \\
\text { Right inserted }\end{array}$}} \\
\hline & \multicolumn{3}{|c|}{ Left uninserted } & \multicolumn{4}{|c|}{ Right inserted } & & & & & & \\
\hline & - & $+\quad++$ & +++ & - & + & ++ & ++++ & - & $+\quad++$ & +++ & - & $+\quad++$ & ++++ \\
\hline \multicolumn{14}{|l|}{ Gingiva } \\
\hline Neutrophilic infiltration ${ }^{\text {a) }}$ & 0 & $\begin{array}{cc}6 & 0 \\
(0.003)\end{array}$ & 0 & 0 & 1 & 2 & 3 & 0 & $\begin{array}{cc}2 & 2 \\
(0.699)\end{array}$ & 3 & 0 & $\begin{array}{cc}3 & 2 \\
(0.058)\end{array}$ & $\begin{array}{l}2 \\
3)\end{array}$ \\
\hline Dilated blood capillaries ${ }^{\mathrm{b})}$ & 3 & $\begin{array}{cc}3 & 0 \\
(0.035)\end{array}$ & 0 & 1 & 1 & 3 & 1 & 2 & $\begin{array}{cc}1 & 4 \\
(0.503)\end{array}$ & 0 & 3 & $\begin{array}{cc}1 & 1 \\
(0.104)\end{array}$ & 1) \\
\hline Swelling of fibroblasts ${ }^{\mathrm{b})}$ & 6 & $\begin{array}{cc}0 & 0 \\
(0.022)\end{array}$ & 0 & 2 & 3 & 1 & 0 & 2 & $\begin{array}{cc}5 & 0 \\
(0.738)\end{array}$ & 0 & 3 & $\begin{array}{cc}2 & 0 \\
(0.313)\end{array}$ & $\begin{array}{l}0 \\
3)\end{array}$ \\
\hline Focal hemorrhage $e^{\text {b) }}$ & 6 & $\begin{array}{cc}0 & 0 \\
(0.341)\end{array}$ & 0 & 5 & 1 & 0 & 0 & 7 & $\begin{array}{cc}0 & 0 \\
(0.300)\end{array}$ & 0 & 5 & $\begin{array}{cc}0 & 0 \\
(0.389)\end{array}$ & $\begin{array}{l}0 \\
9)\end{array}$ \\
\hline Foreign-body granuloma ${ }^{\text {b) }}$ & 5 & 10 & 0 & 5 & 1 & 0 & 0 & 6 & $\begin{array}{cc}1 & 0 \\
(0.915)\end{array}$ & 0 & 2 & $\begin{array}{cc}3 & 0 \\
(0.166)\end{array}$ & $\begin{array}{l}0 \\
5)\end{array}$ \\
\hline \multicolumn{14}{|l|}{ Stratified squamous epithelium } \\
\hline Proliferation ${ }^{\mathrm{c}}$ & 2 & $\begin{array}{cc}4 & 0 \\
(0.002)\end{array}$ & 0 & 0 & 1 & 2 & 3 & 0 & $\begin{array}{cc}2 & 3 \\
(0.478)\end{array}$ & 2 & 0 & $\begin{array}{cc}3 & 2 \\
(0.058)\end{array}$ & 2) \\
\hline Erosion $^{\mathrm{c}}$ & 6 & $\begin{array}{cc}0 & 0 \\
(0.001)\end{array}$ & 0 & 1 & 1 & 4 & 0 & 2 & $\begin{array}{cc}2 & 3 \\
(0.477)\end{array}$ & 0 & 4 & $\begin{array}{cc}1 & 0 \\
(0.013)\end{array}$ & o \\
\hline Edema $^{\text {c) }}$ & 3 & $\begin{array}{cc}3 & 0 \\
(0.006)\end{array}$ & 0 & 0 & 2 & 3 & 1 & 1 & $\begin{array}{cc}5 & 1 \\
(0.045)\end{array}$ & 0 & 2 & $\begin{array}{cc}2 & 1 \\
(0.059)\end{array}$ & 1 \\
\hline \multicolumn{14}{|l|}{ Others } \\
\hline Formation of gingival pocket ${ }^{\mathrm{d})}$ & 6 & $\begin{array}{cc}0 & 0 \\
(0.145)\end{array}$ & 0 & 4 & 2 & 0 & 0 & 3 & $\begin{array}{cc}4 & 0 \\
(0.433)\end{array}$ & 0 & 4 & $\begin{array}{cc}1 & 0 \\
(0.662)\end{array}$ & $\begin{array}{l}0 \\
\text { 2) }\end{array}$ \\
\hline Desquamation of junctional epithelium ${ }^{\mathrm{e}}$ & 6 & $\begin{array}{cc}0 & 0 \\
(0.010)\end{array}$ & 0 & 2 & 0 & 4 & 0 & 1 & $\begin{array}{cc}1 & 5 \\
(0.646)\end{array}$ & 0 & 3 & $\begin{array}{cc}1 & 1 \\
(0.245)\end{array}$ & 1) \\
\hline \multicolumn{14}{|l|}{ Molar } \\
\hline Formation of dental caries ${ }^{\mathrm{e})}$ & 6 & 0 & 0 & 6 & 0 & 0 & 0 & 6 & $\begin{array}{cc}1 & 0 \\
(0.377)\end{array}$ & 0 & 5 & 0 & 0 \\
\hline
\end{tabular}

The numbers of evaluated cases were 6, 7 and 5 in group A, group B and group C, respectively. Differences of experimental samples against the sample of right maxillary site of group A as the basal sample were tested by the unpaired Student's $t$-test. ${ }^{\text {a) }}-$, no changes; +, slight (observed scatteringly); ++, moderate (observed comparatively frequently); +++, severe (observed comparatively frequently with microabscess formation). ${ }^{\text {b) }}-$, no changes; + , slight (observed scatteringly); ++ , moderate (observed omparatively frequently); +++, severe (observed frequently). ${ }^{c)}-$, no changes; + , slight (observed focally); ++, moderate (observed comparatively multifocally); +++, severe (observed multifocally). ${ }^{\text {d) }}-$, no changes; +, slight (small, unilaterally); ++, moderate (small, bilaterally); +++, severe (large). ${ }^{\text {e) }}-$, no changes; +, slight (unilaterally); ++, moderate (bilaterally); +++, severe (not applicable). Statistical $P$ values are given in parentheses.

ous leupeptin treatment in the late stage (the third week to the seventh week after inoculation) of P. gingivalis-induced gingival inflammation has a strong inhibitory effect on the gingival inflammation.

\section{DISCUSSION}

Animal models are critical for elucidating pathogenic processes of periodontal inflammation induced by $P$. gingivalis and repair. In previous studies, oral inoculation with $P$. gingivalis ATCC33277 was unsuccessful even when the microflora was suppressed by means of continuous antibiotic application, using the same inoculation schedule as this experiment. Only weak gingival inflammation was observed and there were no significant histological changes in the periodontal tissues (S. Kitano, unpublished data). Since it has been shown that various strains of $P$. gingivalis had heterogeneous virulence in experimental animal models (21) and that an avirulent, nonpigmented strain of $P$. gingivalis possessed low proteolytic and hemagglutinating activities $(22,23)$, this was thought to be due to a relatively low virulence of this strain in the animal model. In addition, several reports have indicated that protease deficiency in $P$. gingivalis is correlated with virulence $(23-25)$. Therefore, we selected $P$. gingivalis W83 strain as a periodontal pathogen, which is known to be more 

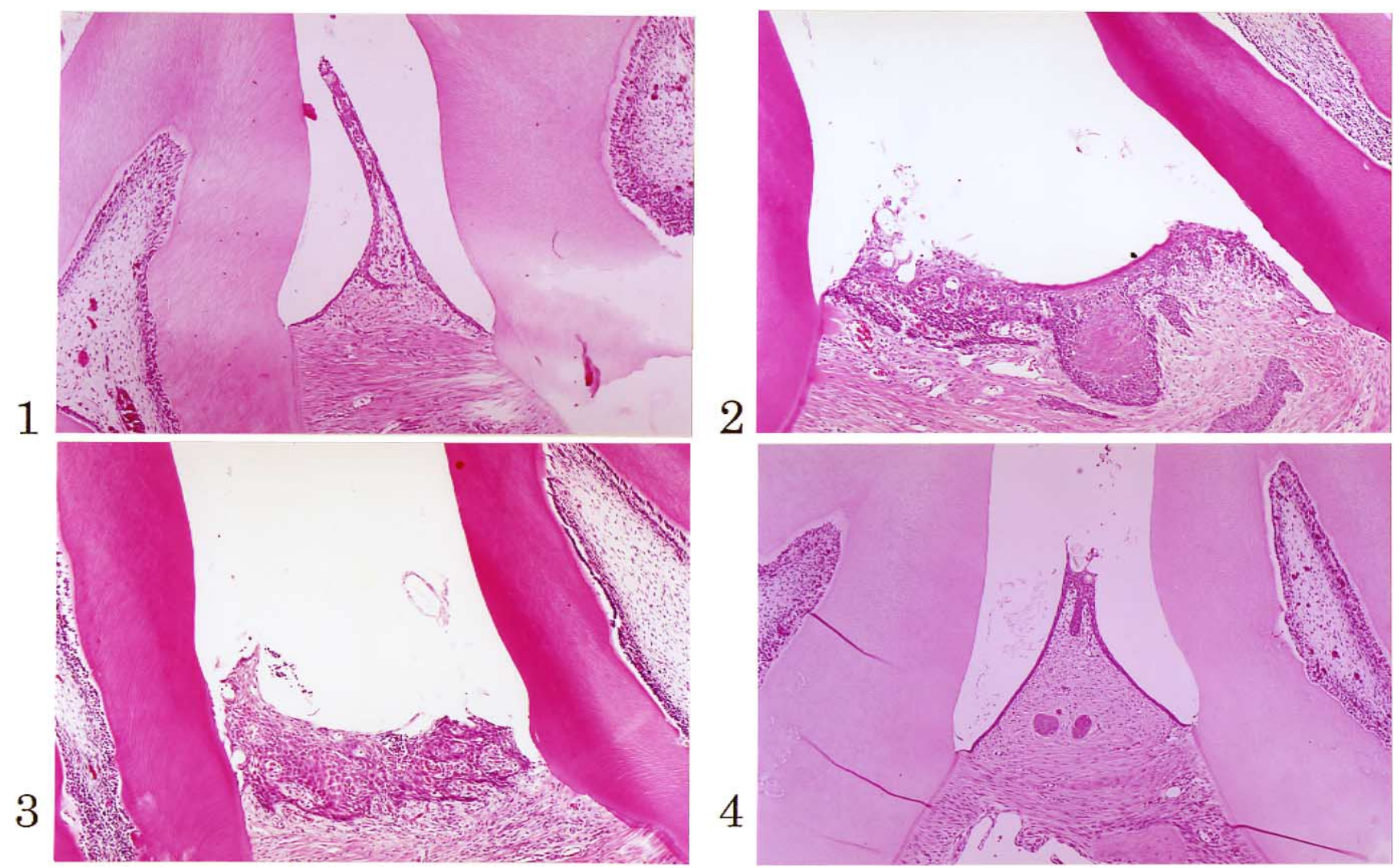

Fig. 3. Comparison of histological sections of the maxillary first and second molars. At 7 weeks after bacterial inoculation, the maxillary bilateral first and second molar samples with periodontal tissues were stained with the hematoxylin-eosin staining solution and examined by light microscopy. Micrographs 1, 2, 3 and 4 show thread-noninserted (left) site in group A, threadinserted site (right) in group A, thread-inserted site (right) in group B and thread-inserted site (right) in group C, respectively. Magnification, $\times 60$.

virulent than the ATCC33277 strain and to exhibit stronger proteolytic activities of Rgp and Kgp than the strain ATCC 33277. The present study indicated that the oral inoculation of this strain for 5 days could successfully cause gingival inflammation in rats. Insertion of the cotton thread between the first and second maxillary molars contributed to adhere the bacterium to the periodontal tissues and to facilitate the induction of gingival inflammation.

The present animal model showed a marked similarity to an infectious process of periodontal diseases in humans and appeared to be suitable for studying specific aspects of gingival inflammation induced by $P$. gingivalis. Clinical landmarks of periodontal infections included a marked neutrophil infiltration, dilated blood capillaries, swelling of fibroblasts, and proliferation, erosion and edema of stratified squamous, and desquamation of junctional epithelium which was essential for the formation of the gingival pockets. However, alveolar bone resorption was not evident. There are several reports on the rat model of experimental periodontal diseases induced by oral inoculation with $P$. gingivalis alone or in a complex with
Streptococcus sanguis, in some of which the alveolar bone loss was induced $(26-30)$. Since, however, the present model showed no significant alveolar bone loss, it was not an animal model of periodontal disease that reproduced all aspects of human periodontitis.

A number of investigations have suggested that two major cysteine proteinases produced by $P$. gingivalis, Rgp and Kgp, play a crucial role in the pathogenesis of progressive periodontal disease in multiple pathways; destruction of periodontal connective tissues, disruption of host defense mechanisms, development and maintenance of inflammation in periodontal pockets (for reviews, see refs. 2-5). With respect to physiology of the bacterium, Rgp and Kgp are indispensable in obtaining peptides/amino acids and heme by the asaccharolytic bacterium and in processing its cell surface and secretory proteins. Given that $P$. gingivalis is a major therapeutic target in the prevention and treatment of periodontal diseases, therefore, the structural and functional studies on Rgp and Kgp are likely to provide important information that will direct the efforts to develop effective therapeutic inhibitors of pathophysiologically relevant 
their proteolytic activities. Leupeptin was a potent inhibitor for Rgp and Kgp, although the latter was slightly less sensitive to this inhibitor than the former $(17,19)$. Thus, the inhibitory effect of leupeptin on $P$. gingivalis-induced gingival inflammation was examined. It is considered that $P$. gingivalis adhered and colonized in periodontal tissue in early stage and that it exhibited the virulence in late stage. We found that this agent had a little inhibitory effect on gingival inflammation when administered in the early stage. However, leupeptin exhibited a strong inhibition on the development and progression of inflammation in the late stage when it was administered for the period of 40 days after the last inoculation of $P$. gingivalis (Table 2 and Fig. 3), indicating that this agent is more effective in the late stage of inflammation. These results provided evidence that Rgp and Kgp play a major role in the development and progression of $P$. gingivalis-induced gingival inflammation in rats and that proteinase inhibitors for Rgp and Kgp may provide a means of preventing periodontal diseases.

\section{REFERENCES}

1 Slot J, Bragd L, Wilkstrom M and Dahlen G: The occurrence of Actinobacillus actinomycetemcomitance, Bacteroides gingivalis and Bacteroides intermedius in destructive periodontal disease in adults. J Clin Periodontol 13, 576 - 577 (1986)

2 Yamamoto K, Kadowaki T, Okamoto K, Abe N and Nakayama $\mathrm{K}$ : Biological roles of a novel class of cysteine proteinases from Porphyromonas gingivalis in periodontal disease progression. In Medical Aspects of Proteases and Protease Inhibitors, Edited by Katunuma N, Kido H, Fritz H and Travis J, pp 139 - 149, IOS Press, Amsterdam (1997)

3 Travis J, Pike R, Imamura T and Potempa J: Porphyromonas gingivalis proteinases as virulence factors in the development of periodontitis. J Periodont Res 32, 120 - 125 (1997)

4 Yamamoto K, Kadowaki T and Okamoto K: Arg-gingipain and Lys-gingipain: a novel class of cysteine proteinases, In Proteases: New Perspectives, Edited by Turk V, pp 175-184, Birkhäuser Verlag, Basel (1999)

5 Kadowaki T, Nakayama K, Okamoto K, Abe N, Baba A, Shi Y, Ratnayake DB and Yamamoto K: Porphyromonas gingivalis proteinases as virulence determinants in progression of periodontal diseases. J Biochem 128, 153 - 159 (2000)

6 Shi Y, Ratnayake DB, Okamoto K, Abe N, Yamamoto K and Nakayama K: Genetic analysis of proteolysis, hemoglobin binding, and hemagglutination of Porphyromonas gingivalis: construction of mutants with a combination of $\operatorname{rgpA}, \operatorname{rgpB}, \mathrm{kgp}$, and hagA. J Biol Chem 274, 17955 - 17960 (1999)

7 Potempa J, Pike R and Travis J: The multiple forms of trypsinlike activity present in various strains of Porphyromonas gingivalis are due to the presence of either arg-gingipain or lys-gingipain. Infect Immun 63, 1176 - 1182 (1995)

8 Wingrove JA, DiScipio RG, Chen Z, Potempa J, Travis J and Hugli TE: Activation of complement components C3 and C5 by a cysteine proteinase (Gingipain-1) from Porphyromonas (Bacteroides) gingivalis. J Biol Chem 267, 18902-18907 (1992)
9 Kadowaki T, Yoneda M, Okamoto K, Maeda K and Yamamoto $\mathrm{K}$ : Purification and characterization of a novel arginine-specific cysteine proteinase (argingipain) involved in the pathogenesis of periodontal disease from the culture supernatant of Porphyromonas gingivalis. J Biol Chem 269, 21371-21378 (1994)

10 Nakayama K, Kadowaki T, Okamoto K and Yamamoto K: Construction and characterization of arginine-specific cysteine proteinase (Arg-gingipain)-deficient mutants of Porphyromonas gingivalis. J Biol Chem 270, 23619 - 23626 (1995)

11 Abe N, Kadowaki T, Okamoto K, Nakayama K, Ohishi M and Yamamoto K: Biochemical and functional properties of lysinespecific cysteine proteinase (Lys-gingipain) as a virulence factor of Porphyromonas gingivalis in periodontal disease. J Biochem 123, 305-312 (1998)

12 Scott CF, Whitaker EJ, Hammond BF and Colman RW: Purification and characterization of a potent $70-\mathrm{kDa}$ thiol lysyl-proteinase (Lys-gingivain) from Porphyromonas gingivalis that cleaves kininogens and fibrinogen. J Biol Chem 268, $7935-$ 7942 (1993)

13 Imamura T, Potempa J, Pike RN, Moore JN, Barton MH and Travis J: Effect of free and vesicle-bound cysteine proteinases of Porphyromonas gingivalis on plasma clot formation: implications for bleeding tendency at periodontitis sites. Infect Immun 63, 4877 - 4882 (1995)

14 Okamoto K, Nakayama K, Kadowaki T, Abe N, Ratnayake DB and Yamamoto K: Involvement of a lysine-specific cysteine proteinase in hemoglobin adsorption and heme accumulation by Porphyromonas gingivalis. J Biol Chem 273, $21225-21231$ (1998)

15 Imamura T, Pike RN, Potempa $\mathrm{J}$ and Travis $\mathrm{J}$ : Pathogenesis of periodontitis: a major arginine-specific cysteine proteinase from Porphyromonas gingivalis induces vascular permeability enhancement through activation of the kallikrein/kinin pathway. J Clin Invest 94, 361 - 367 (1994)

16 Fletcher HM, Schenkein HA, Morgan RM, Bailey KA, Berry CR and Macrina FL: Virulence of a Porphyromonas gingivalis W83 mutant defective in the prt $\mathrm{H}$ gene. Infect Immun 63, 1521 - 1528 (1995)

17 Kadowaki T, Nakayama K, Yoshimura F, Okamoto K, Abe N and Yamamoto $\mathrm{K}$ : Arg-gingipain acts as a major processing enzyme for various cell surface proteins in Porphyromonas gingivalis. J Biol Chem 273, 29072 - 29076 (1998)

18 Baker PJ, Evans RT and Roopenian DC: Oral infection with Porphyromonas gingivalis and induced alveolar bone loss in immunocompetent and severe combined immunodeficient mice. Archs Oral Biol 39, 1035 - 1040 (1994)

19 Abe N, Kadowaki T, Okazaki S, Asao T and Yamamoto K: A sensitive assay for Lys-gingipain from Porphyromonas gingivalis using new synthetic substrates. Jpn J Pharmacol 82, Suppl I, 266P (2000)

20 Japan Society of Chemotherapy: Methods for the determination of minimum inhibitory concentration (MIC) by microdilution method. Chemotherapy (Tokyo) 41, 183 - 189 (1993)

21 Neider ME, Chen PB, Suido H, Reynolds HS, Zambon JJ, Shlossman M and Genco RJ: Heterogeneity of virulence among strains of Bacteroides gingivalis. J Periodont Res 24, $192-198$ (1989)

22 Mckee AS, McDermid AS, Wait R, Baskerville A and Marsh PD: Isolation of colonial variants of Bacteroides gingivalis W50 
with a reduced virulence. J Med Microbiol 27, 59 - 64 (1988)

23 Shah HN, Seddon SV and Gharbia SE: Studies on the virulence properties and metabolism of pleiotropic mutants of Porphyromonas gingivalis (Bacteroides gingivalis) W50. Oral Microbiol Immunol 4, 19 - 23 (1989)

24 van Steenbergen TJM, Delemarre FGA, Namavar F and DeGraaff J: Differences in virulence within the species Bacteroides gingivalis. Antonie Van Leeuwenhoek 53, $233-$ 244 (1987)

25 Kesavalu L, Ebersole JL, Machen RL and Holt SC: Porphyromonas gingivalis virulence in mice: induction of immunity to bacterial components. Infect Immun 60, 1455 1464 (1992)

26 Evans RT, Klausen B, Ramamurthy NS, Golub LM, Sfintescu C and Genco RJ: Periodontopathic potential of two strains of Porphyromonas gingivalis in gnotobiotic rats. Arch Oral Biol 37, 813 - 819 (1992)

27 Golub LM, Evans RT, McNamara TF, Lee HM and Ramamur- thy NS: A non-antimicrobial tetracycline inhibits gingival matrix metalloproteinases and bone loss in Porphyromonas gingivalisinduced periodontitis in rats. Ann NY Acad Sci 732, 96-111 (1994)

28 Klausen B, Evans RT, Ramamurthy NS, Golub LM, Sfintescu C, Lee JY, Bedi G, Zambon JJ and Genco RJ: Periodontal bone level and gingival proteinase activity in gnotobiotic rats immunized with Bacteroides gingivalis. Oral Microbiol Immunol 6, $193-201$ (1991)

29 Chang KM, Ramamurthy NS, McNamara TF, Evans RT, Klausen B, Murray PA and Golub LM: Tetracyclines inhibit Porphyromonas gingivalis-induced alveolar bone loss in rats by a non-antimicrobial mechanism. J Periodontal Res 29, 242 - 249 (1994)

30 Fiehn NE, Klausen B and Evans RT: Periodontal bone loss in Porphyromonas gingivalis-infected specific pathogen-free rats after preinoculation with endogenous Streptococcus sanguis. J Periodontal Res 27, 609-614 (1992) 